\title{
Perfil dos recém-nascidos submetidos à correção cirúrgica de persistência do canal arterial à beira do leito
}

\section{Profile of newborns who underwent surgical correction of patent ductus arteriosus at the bedside}

Camila Menezes Rabelo ${ }^{1}$. Valdester Cavalcante Pinto Junior². José Eloy da Costa Filho ${ }^{3}$. Eveline Campos Monteiro de Castro ${ }^{4}$. Simone da Silveira Magalhães Aragão ${ }^{5}$.

1 Médica Pediatra e Neonatologista da Maternidade Escola Assis Chateaubriand (MEAC), Fortaleza, Ceará, Brasil. 2 Mestre em Avaliação de Políticas Públicas, Médico cirurgião cardiovascular do Hospital Universitário Walter Cantídio (HUWC), Fortaleza, Ceará, Brasil. 3 Médico Cardiologista e Ecocardiografista da Maternidade Escola Assis Chateaubriand (MEAC), Fortaleza, Ceará, Brasil. 4 Doutora em Pediatria pela Universidade Federal de São Paulo (UNIFESP), Médica Pediatra e Neonatologista, Chefe da Unidade Neonatal da Maternidade Escola Assis Chateaubriand (MEAC), Fortaleza, Ceará, Brasil. 5 Mestre em Cuidados Clínicos em Enfermagem e Saúde, Enfermeira do centro cirúrgico do Hospital Universitário Walter Cantídio (HUWC), Fortaleza, Ceará, Brasil.

\section{RESUMO}

Introdução: malformações congênitas são importantes causas de mortalidade infantil nos recém-nascidos (RNs) prematuros, sendo a cardiopatia congênita (CC) uma das responsáveis. Objetivo: demonstrar o perfil clínico e epidemiológico dos RNs submetidos à cirurgia de correção de persistência do canal arterial (PCA) à beira do leito. Material e Métodos: estudo longitudinal retrospectivo, realizado nas Unidades de Terapia Intensiva Neonatal de um hospital terciário, no período de janeiro a dezembro de 2015. Resultados: a maioria dos pacientes foram RNs de extremo baixo peso e do sexo feminino, que apresentaram as mais variadas manifestações clínicas e complicações como hemorragia peri-intraventricular, insuficiência real e displasia broncopulmonar, mesmo tendo feito uso de corticoide antenatal, surfactante e suporte ventilatório. Conclusão: RNs submetidos à cirurgia foram àqueles prematuros extremos, do sexo feminino e extremo baixo peso ao nascer, que sofreram com complicações maternas ainda no período gestacional.

Palavras-chave: Canal arterial. Prematuro. Tratamento medicamentoso. Cirurgia.

\section{ABSTRACT}

Introduction: congenital malformations are important causes of infant mortality in preterm infants, and congenital heart disease one of the responsible. Objective: demonstrate the clinical and epidemiological profile of newborns undergoing persistence correction surgery ductus arteriosus at the bedside. Methods: retrospective longitudinal study, performed at Neonatal Intensive Care Units of tertiary hospital, between January and December 2015. Results: most patients were infants of extremely low birth weight and female, who presented the most varied clinical manifestations and complications such as peri-intraventricular hemorrhage, renal failure and bronchopulmonary dysplasia, even having made use of corticosteroids antenatal, surfactant and ventilator support. Conclusion: newborns undergoing surgery were those extremely premature infants, women and extremely low birth weight, which suffered from maternal complications during pregnancy yet.

Keywords: Ductus arteriosus. Infant, premature. Drug therapy. Surgery.

Autor correspondente: Camila Menezes Rabelo, Rua Gonçalves Ledo, n 10, apartamento 1709, Praia de Iracema, Fortaleza, Ceará. CEP: 60060325.Telefone: +55 85 99928-0627. E-mail: mdcamila@hotmail.com

Conflito de interesses: Não há qualquer conflito de interesses por parte de qualquer um dos autores.

Recebido em: 21 Abr 2016; Revisado em: 23 Mai 2016; Aceito em: 23 Mai 2016. 


\section{INTRODUÇÃO}

Melhorias nos cuidados neonatais têm contribuído para aumentar a sobrevida dos recém-nascidos prematuros de muito baixo peso ao nascer e, consequentemente, reduzido significativamente a mortalidade perinatal. ${ }^{1-3}$ Podendo ainda se observar nesse grupo de pacientes frequentes complicações, internações prolongadas e sequelas, sendo fatores determinantes para dados da morbimortalidade. ${ }^{1,4,5}$

As malformações congênitas continuam a ser importantes causas de mortalidade infantil nessa faixa etária, sendo a cardiopatia congênita (CC) uma das responsáveis por esses resultados. ${ }^{6-8} \mathrm{O}$ diagnóstico da doença cardíaca é maior para a população de terapia intensiva neonatal, dado que o baixo peso ao nascer e bebês pequenos para a idade gestacional são fatores de alto risco, ${ }^{9}$ com prevalência de $10,7 \%$ a 40,7\% dos nascimentos. ${ }^{10}$

A persistência do canal arterial (PCA) ou do ducto arterioso (PDA) refere-se à manutenção do canal arterial após 72 horas de vida. ${ }^{11}$ Este corresponde a um conduto vascular que comunica a circulação sistêmica à pulmonar, ligando aorta descendente e artéria pulmonar. ${ }^{12}$

Em recém-nascidos de termo, o fechamento do canal arterial ocorre em até três dias de vida. ${ }^{13-15}$ Já no prematuro, esse fechamento é retardado devido à imaturidade do ducto, resultando em efeitos nocivos sobre o crescimento e desenvolvimento normais. ${ }^{12,16,17}$

O quadro clínico está relacionado com o calibre do canal arterial e, consequentemente, com o shunt esquerda-direita. ${ }^{12,18}$ Devido à resistência pulmonar elevada nos primeiros dias de vida, os sinais clínicos podem ser retardados inicialmente. ${ }^{18}$ Assim, taquicardia, sopro cardíaco sistólico, precórdio dinâmico, pulsos amplos e hemorragia pulmonar (HP) passam a ser evidentes logo após as primeiras $72 \mathrm{~h}$ ou após administração de surfactante. ${ }^{12,14,17,19}$ A persistência do canal arterial pode ser responsável por consequências importantes como insuficiência cardíaca congestiva (ICC), displasia broncopulmonar (DBP), enterocolite necrozante (ECN) e hemorragia periintraventicular (HPIV), condições que resultam do desvio de fluxo sistêmico para o pulmão. ${ }^{11,12,20}$

Restrição hídrica, diuréticos e inotrópicos para tratar a insuficiência cardíaca não são suficientes para o canal com repercussão hemodinâmica. ${ }^{12,13,21}$ Associado a essas medidas, muitos centros optam pela administração de inibidores da ciclooxigenase (indometacina e ibuprofeno), bem como por um suporte ventilatório adequado. ${ }^{11,21,22}$

Para os recém-nascidos que não respondem positivamente ou não podem ser submetidos às técnicas conservadoras, a abordagem cirúrgica do canal arterial é uma opção. ${ }^{12,16,21,23}$ No entanto, o ideal momento para a sua realização ainda é uma questão controversa. ${ }^{12,21,24}$

A relevância desse estudo está em demonstrar o perfil clínico e epidemiológico dos recém-nascidos submetidos à cirurgia de correção de persistência do canal arterial à beira do leito, bem como a melhor hora para sua realização.

\section{MATERIAL E MÉTODOS}

Trata-se de um estudo longitudinal retrospectivo, documental, realizado na Unidade Neonatal da Maternidade Escola Assis Chateaubriand (MEAC) da Universidade Federal do Ceará (UFC), instituição terciária de referência para gravidezes de risco no município de Fortaleza, Ceará.

Foram eleitos para participar do estudo todos os recémnascidos submetidos à cirurgia de correção de persistência do canal arterial à beira do leito, no período de janeiro de 2015 a dezembro de 2015. A amostra foi intencional, quando o pesquisador decide propositalmente sobre a seleção de sujeitos considerados típicos da população. ${ }^{25}$

Para a realização da coleta de dados foi utilizado formulário estruturado, para obter informações, que foram colhidas diretamente de registros em prontuários. Os dados coletados se referem às variáveis maternas, aos dados perinatais e características clínicas.

Variáveis maternas foram: idade materna, sendo considerada aquela registrada na data do parto; história gestacional, que inclui complicações da gestação atual, como síndrome hipertensiva, diabetes gestacional e infecção.

Dentre as variáveis perinatais: idade gestacional, calculada pela data da última menstruação ou informações pelo ultrassom precoce (anterior a 20 semanas de gestação). Quando essas informações não estavam disponíveis, utilizou-se ultrassom tardio, juntamente com exame clínico neurológico do recém-nascido (New Ballard); recém-nascido prematuro, aquele cujo nascimento ocorreu com idade gestacional inferior a 37 semanas completas de gestação; gênero, definido como masculino ou feminino no primeiro exame físico do recém-nascido; peso de nascimento, obtido por meio de procedimento realizado por profissional habilitado (médico ou enfermeiro) em sala adequada para os primeiros cuidados do neonato, logo após o parto, com criança completamente despida (utilizada balança eletrônica pediátrica devidamente tarada); uso de corticoide antenatal, informação obtida por meio de consulta ao prontuário, sendo considerado como uso qualquer tratamento efetuado, completo ou não; uso de surfactante pós-natal (surfactante exógeno na dose de $100 \mathrm{mg} / \mathrm{kg}$ ) em recém-nascidos com sinais de desconforto respiratório importante, em uso de suporte ventilatório.

As variáveis clínicas observadas durante internamento hospitalar foram persistência do canal arterial, displasia broncopulmonar (necessidade de oxigenação às 36 semanas de idade gestacional e com a evolução da doença pulmonar a longo prazo), hemorragias pulmonar e peri-intraventricular, enterocolite necrozante, insuficiências cardíacas (definida 
pela presença de sinais e sintomas, como taquicardia, cardiomegalia, sudorese, oligúria, taquipneia e, até mesmo, dificuldade na mamada, que ocorrem quando o coração é incapaz de suprir as demandas metabólicas dos tecidos) e renal (definida como deterioração aguda da capacidade dos rins em manter a homeostasia dos fluidos corporais por uma diminuição da taxa de filtração glomerular, que determina um acúmulo de água e de solutos, tais como: ureia, creatinina, potássio, sódio e íons hidrogênio).

Quanto aos métodos diagnósticos de imagem foram encontradas alterações radiográficas relacionadas à trama vascular pulmonar. O ecocardiograma bidimencional com Doppler e mapeamento de fluxo em cores, foi realizado utilizando-se o aparelho TOSHIBA XARIO XG ISTYLE, com transdutor específico para recém-nascido (PST-65 AT 6,5 mHz) e foi considerado com repercussão hemodinâmica aquele com relação átrio esquerdo/aorta $>1,5 \mathrm{~mm}$, fluxo reverso em aorta descendente, fluxo reverso na artéria mesentérica superior ou índice de resistência $>0,8$.

Os dados coletados foram organizados em banco de dados no programa Windows Excel, versão 11.0 e processados por programa estatístico SPSS versão 20.0 (Statical Package For The Social Sciences). A análise dos dados foi realizada de forma descritiva, usando as frequências (absoluta e percentual) e as medidas paramétricas (média, mediana e desvio padrão). Os resultados foram agrupados e expostos em gráficos ou tabelas.

Foram seguidos todos os princípios éticos para pesquisa com seres humanos respeitando a resolução 466/2012 do Conselho Nacional de Saúde. ${ }^{26}$ O projeto de pesquisa foi aprovado, sem restrições, com CAAE:54407216.70000.5050 pelo Comitê de Ética em Pesquisa da Maternidade Escola Assis Chateaubriand/ MEAC/UFC.

\section{RESULTADOS}

No período de janeiro de 2015 a dezembro de 2015, na MEAC-UFC nasceram um total de 5477 recém-nascidos dos quais, $1165(21,2 \%)$ eram prematuros com idade gestacional $<37$ semanas.

No grupo selecionado para este trabalho foram incluídos todos os recém-nascidos submetidos à cirurgia de correção de persistência do canal arterial à beira do leito em Unidade de Terapia Intensiva neonatal de maternidade de referência terciária. Chegou-se a uma amostra final de 14 recém-nascidos submetidos ao procedimento cirúrgico.

A idade materna variou de 15 a 36 anos com média de 23,8 ( \pm 7,49 anos). O percentual de gestações em mães com idade igual ou superior a 35 anos foi de $14,2 \%$ e $42,8 \%$ foram em mães adolescentes ( $\leq 20$ anos). Levando-se em consideração complicações maternas durante a gestação destacou-se síndrome hipertensiva, diabetes gestacional e infecção, conforme Tabela 1.
Tabela 1. Complicações maternas.

Síndrome hipertensiva (n, \%)

SIM

NÃO

$8(57,2 \%)$

Diabetes gestacional (n, \%)

SIM

0

NÃO

$14(100 \%)$

Infecção perinatal (n, \%)

SIM

$9(64,2 \%)$

NÃO

dp:desvios padrão; n: número da amostra; \%:porcentagem da amostra

A maioria da população foi do sexo feminino $(57,1 \%)$, com peso de nascimento variando entre $650 \mathrm{~g}$ e $1370 \mathrm{~g}$ (média de $815 \mathrm{~g} \pm 175,5 \mathrm{~g}$ ). A maior parcela dos pacientes foram recém-nascidos de extremo baixo peso, inferior a $1000 \mathrm{~g}$ $(92,8 \%)$ e a idade gestacional variou de 23 s6d a 30 s $3 \mathrm{~d}$ com média de $26 \mathrm{~s} 1 \mathrm{~d}$ ( $\pm 12,3$ dias), sendo $78,5 \%$ prematuros com idade gestacional inferior a 28 semanas.

Em relação às medidas adotadas pela instituição evidenciouse uso de corticoide antenatal em $85,7 \%$, surfactante e suporte de ventilação mecânica invasiva em $100 \%$ e restrição hídrica (<80 ml/kg/dia) em 92,8\%.

Dos 14 casos estudados, manifestações clínicas variadas foram observadas, dentre elas: taquicardia, sopro cardíaco, desconforto respiratório e congestão pulmonar por hiperfluxo Gráfico 1. Já em relação às complicações a longo prazo pôde-se ver hemorragia pulmonar, hemorragia peri-intraventricular, insuficiência renal, insuficiência cardíaca, displasia broncopulmonar e enterocolite necrosante - Tabela 2 .

Gráfico 1. Apresentação clínica nos neonatos.

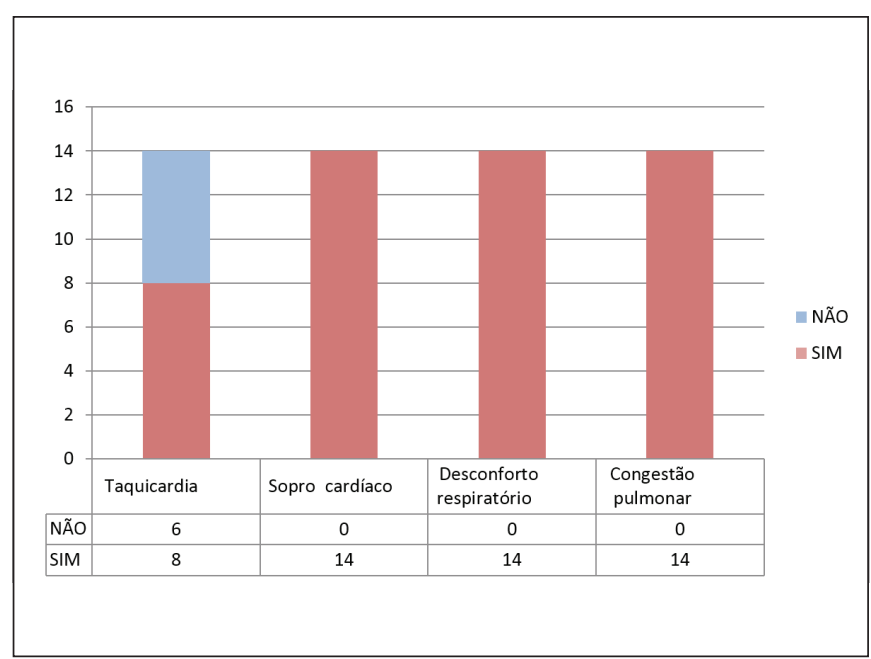


Tabela 2. Complicações nos recém-nascidos.

Displasia broncopulmonar (n,\%)

SIM

$14(100 \%)$

NÃO

Hemorragia pulmonar (n,\%)
SIM
$5(35,7 \%)$
NÃO
$9(64,3 \%)$

Hemorragia peri-intraventricular (n,\%)

SIM

$13(92,8 \%)$

NÃO

Enterocolite necrozante (n,\%)

SIM

NÃO

Insuficiência renal aguda ( $\mathrm{n}, \%)$

SIM

NÃO

Insuficiência cardíaca (n,\%)

SIM

NÃO

n: número da amostra

A idade dos recém-nascidos no momento do procedimento cirúrgico variou de 18 a 68 dias de vida, sendo a média de 31 dias de vida $(\mathrm{dp}= \pm 13,18)$. Já no que diz respeito ao sucesso cirúrgico, todos os recém-nascidos submetidos à cirurgia de correção de PCA sobreviveram.

\section{DISCUSSÃO}

Os neonatos prematuros assistidos na Unidade Neonatal da Maternidade Escola Assis Chateaubriand - UFC foram abordados desde a implantação do protocolo de assistência, cuidados diagnósticos e terapêuticos, com registro de dados em prontuários e posterior coleta.

A persistência do canal arterial nos recém-nascidos prematuros é um evento multifatorial que tem sido objeto de inúmeros estudos nos últimos anos e que é responsável por repercussões clínicas e hemodinâmicas de grande significância na morbidade e mortalidade desses pacientes. Representa 12\% das cardiopatias congênitas acianogênicas, sendo assim a mais comum em crianças. ${ }^{14,27,28}$ Nos recém-nascidos prematuros, sua incidência é inversamente proporcional à idade gestacional e ao peso. Nos com peso de nascimento inferior a $1000 \mathrm{~g}$, aumenta a incidência para 70 a $80 \% .^{27}$

A prematuridade e o extremo baixo peso ao nascer são fatores de risco relacionados à persistência do canal arterial. Esse dado pôde ser confirmado por vários autores em suas pesquisas sobre o assunto, nos quais a prevalência de canal arterial, hemodinamicamente significativo, foi bem maior nos recém-nascidos com peso de nascimento inferior a $1000 \mathrm{~g}$ e idade gestacional menor que 30 semanas. ${ }^{29} \mathrm{O}$ estudo evidenciou também que a maioria da população era composta por prematuros de extremo baixo peso ao nascer.

Assim como em outras publicações, evidenciamos maior prevalência no sexo feminino.

De acordo com a literatura, idade materna, história gestacional e via de parto não se relacionam com uma maior frequência de recém-nascidos com persistência do canal arterial. ${ }^{14,27}$

A administração antenatal de corticóide é rotina recomendada a todas as gestantes com trabalho de parto prematuro antes de 34 semanas de idade gestacional. Seu papel na maturação pulmonar fetal e neonatal já está bem estabelecido, uma vez que atua diretamente na parede ductal com redução da resposta vasodilatadora das prostaglandinas e, por isso, é aceito como fator protetor na prevalência do canal arterial patente em prematuros. ${ }^{9,12,30}$

O fluxo sanguíneo pelo canal arterial patente (fluxo esquerda-direita) está diretamente relacionado ao seu diâmetro e, por conseguinte, à clínica do recém-nascido. ${ }^{14,17}$ Através do ducto uma parcela do débito ventricular esquerdo é desviada à artéria pulmonar na sístole, e no movimento de diástole, ocorre um desvio de fluxo da aorta para vasos pulmonares. ${ }^{14,17,18}$ O sangue desviado ao pulmão gera uma redução do fluxo sanguíneo sistêmico, aumentando a velocidade de fluxo na artéria pulmonar, o que pode ser responsável pela hemorragia pulmonar e pelo desenvolvimento de displasia broncopulmonar em alguns casos. ${ }^{3,18,19}$

A existência de doença pulmonar associada, como síndrome do desconforto respiratório, dificulta a avaliação diagnóstica. Isso se justifica, pois nos primeiros dias de vida, a resistência pulmonar encontra-se elevada e, após $72 \mathrm{~h}$ ou uso de surfactante, há uma piora nos sinais de desconforto respiratório. O shunt esquerda-direita através do canal, que ocorre em consequência à redução da resistência vascular pulmonar, causa aumento do fluxo sanguíneo pulmonar sendo responsável por importante edema no pulmão e piora da ventilação nesses pacientes. . $^{12,14,17,18}$

Nos primeiros dias de vida, pode-se observar quadro de taquicardia e insuficiência cardíaca causada por uma sobrecarga das câmaras cardíacas esquerdas pela presença de amplo canal arterial. Contribuindo ainda para piora do quadro de desconforto respiratório já existente. O sopro cardíaco é sistólico e pode ser observado após as primeiras $48 \mathrm{~h}$ de vida, tendo acurácia limitada, pois tem especificidade moderada e sensibilidade baixa nos casos de PCA significativo ao ecocardiograma. ${ }^{14,15,17,19}$

O roubo do fluxo sistêmico, que pode chegar até $1 / 3$ do débito cardíaco, torna-se responsável por um déficit importante na circulação sistêmica causando repercussão clínica nos mais variados órgãos dependentes de uma boa nutrição sanguínea. ${ }^{17,18}$ São comprometidos os fluxos na aorta abdominal, artérias 
renais e mesentéricas, artérias carótidas e artéria cerebral média. Alteração na velocidade do fluxo sanguíneo cerebral é responsável pela hemorragia peri-intraventricular, déficit no plexo mesentérico aumenta o risco de enterocolite necrosante, e no que diz respeito aos rins, quadros graves de insuficiência renal são encontrados e entram como contraindicação ao tratamento clínico do canal arterial patente. A disfunção renal apresenta resposta importante após o fechamento do canal, com melhora da diurese e redução das escórias urinárias. ${ }^{3,17-19}$

No que diz respeito às primeiras condutas a serem tomadas quando existe confirmação de persistência de canal arterial em recém-nascido prematuro é em relação ao tratamento convencional da insuficiência cardíaca congestiva. Medidas como restrição hídrica, diuréticos, oxigenação adequada e inotrópicos são consideradas. No entanto, deve-se ter em mente que elas não fazem parte do tratamento para o canal arterial patente, podendo atrasar o tratamento específico. ${ }^{12,19,24}$ O uso de furosemida deve ser evitado nesses casos, pois é responsável pelo aumento da produção de prostaglandina E2 renal, reduzindo a possibilidade de fechamento espontâneo do canal. ${ }^{12,13,22}$

O fechamento espontâneo do canal, bem como a indicação e o tipo de tratamento a ser instituído em cada recém-nascido prematuro são motivos de inúmeras discussões nos tempos atuais. ${ }^{14,17}$ Optando-se por conduta clínica, recomenda-se o uso de inibidores da síntese de prostaglandinas (indometacina ou ibuprofeno), os quais mostram eficácia maior, com necessidade de menores doses, no fechamento do canal quando usados na primeira semana pós-natal. Os anti-inflamatórios são inibidores seletivos da ciclooxigenase, as quais são responsáveis pela conversão de ácido araquidônico em precursores

\section{REFERÊNCIAS}

1. Lemons JA, Bauer CR, Oh W, Korones SB, Papile LA, Stoll BJ, et al. Very low birth weight outcomes of the National Institute of Child health and Human Development Neonatal Research Network, January 1995 through December 1996. Pediatrics. 2001;107(1):1-8.

2. Hintz SR, Poole WK, Wright LL, Fanaroff AA, Kendrick DE, Laptook AR, et al. Changes in mortality and morbidities among infants born at less than 25 weeks during the post-surfactant era. Arch Dis Child Fetal Neonatal Ed. 2005;90(2):F128-33.

3. Whitfield J, Charsha D, Sprague P. In search of excellence - the Neonatal Intensive Care Quality Improvement Collaborative. BUMC Proceedings [Internet]. 2001[acesso em: 20 Fev 2016];14(1):947 Disponível em: http://www.baylorhealth.edu/Documents/ BUMC\%20Proceedings/2001\%20Vol\%2014/No.\%201/14_1_\%20 Whitfield.pdf

4. Almeida MF, Guinsburg R, Martinez FE, Procianoy RS, Leone $\mathrm{CR}$, Marba ST, et al. Fatores perinatais associados ao óbito precoce em prematuros nascidos nos centros da Rede Brasileira de Pesquisas Neonatais. J Pediatric (Rio J). 2008;84(4):300-7.

5. Itabashi K, Horiuchi T, Kusuda S, Kabe K, Itani Y, Nakamura T, et al. Mortality rates for extremely low birth weight infants born in Japan in 2005. Pediatrics. 2009;123(2):445-50. das prostaglandinas e, assim, atuando no fechamento do canal arterial patente. ${ }^{13,22}$ No que diz respeito aos riscos e benefícios das duas medicações, estudos atuais concluíram que o ibuprofeno é tão eficaz quanto a indometacina, não havendo diferenças significativas no risco de hemorragia peri-intraventricular ou displasia broncopulmonar e nem na mortalidade. No entanto, observou-se maiores complicações renais e enterocolite necrosante nos recém-nascidos tratados com indometacina. ${ }^{13,18,22,24}$

O tratamento, seja clínico ou cirúrgico, só deve ser instituído naqueles neonatos em que a presença do canal arterial esteja causando repercussão hemodinâmica. ${ }^{10,24} \mathrm{~A}$ abordagem cirúrgica ainda é questão de bastante controvérsia e questionada, bem como, o melhor momento para sua realização. A cirurgia para ligadura de canal tem sido utilizada como opção terapêutica naqueles casos em que houve falha ou contraindicação ao tratamento medicamentoso. ${ }^{5,16,21}$ Assim como na literatura, os prematuros encaminhados para cirurgia à beira do leito foram aqueles com falha no tratamento clínico, apresentando bom resultado cirúrgico.

\section{CONCLUSÃO}

Conclui-se, portanto, que o perfil das crianças das Unidades de Cuidados Neonatais da Maternidade Escola Assis Chateaubriand submetidas à correção cirúrgica de PCA são de recém-nascidos prematuros extremos, do sexo feminino e de extremo baixo peso ao nascer, que em algum período da gestação sofreram com complicações maternas, seja hipertensão ou infecção, e que na sua maioria foram submetidos ao uso antenatal de corticoide, surfactante pós-natal e ventilação mecânica invasiva.

6. Duff DF, McNamara DG. History and physical examination of the cardiovascular system. In: Garson A Jr, Bricker TM, Fisher DJ, Neish SR. The science and practice of pediatric cardiology. 2nd ed. Baltimore: Williams \& Wilkins; 1998. p. 693-713.

7. Report of the New England Regional Infant Cardiac Program. Pediatrics. 1980;65(2):375-461.

8. Reller MD, Strickland MJ, Riehle-Colarusso T, Mahle WT, Correa A. Prevalence of congenital heart defects in metropolitan Atlanta, 1998-2005. J Pediatr. 2008;153(6)-807-13.

9. McGrath JM. Early detection and immediate management of congenital heart disease is important to long-term outcomes. J Perinat Neonatal Nurs. 2006;20(4):285-6.

10. Sadeck LS, Azevedo R, Barbato AJ, Calil VM, Latorre MR, Leone $\mathrm{CR}$, et al. Indicações clínico-epidemiológicas para investigação ecocardiográfica no período neonatal: valor dos grupos de risco. Arq Bras Cardiol. 1997;69(5):301-7.

11. Popat H, Kapoor V, Travadi J. Patent Ductus Arteriosus in Infants $<29$ weeks Gestation - Outcomes and Factors Affecting Closure. Indian Pediatr. 2012;49(8):615-20.

12. Margotto PR. Assistência ao recém-nascido de risco. 3 ed. 
Brasília: Pórfiro; 2013. Capítulo 8, Persistência da Canal Arterial; p. 287-93.

13. Cooke L, Steer PA, Woodgate PG. Indomethacin for asymptomatic patent ductus arteriosus in preterm infants. Cochrane Database Syst Rev. 2003;(2):CD003745.

14. Moore P, Brook MM. Patent dusctus arteriosus and Aortopulmonary Window. In: Allen HD, Driscoll DJ, Shaddy RE, Feltes TF. Heart Disease in Infants, Children and Adolescents: including the fetus and young adults. 8th ed. Baltimore: Williams and Wilkins; 2013. p. 722.

15. Huhta JC. Patent ductus arteriosus in the preterm neonate. In Long WA. Fetal and Neonatal Cardiology. Philadelphia: WB Sauders; 1990. p. 389-400.

16. Cho J, Yoon YH, Kim JT, Kim KH, Lim HK, Jun YH, et al. Patent ductus arteriosus closure in prematurities weighing less than $1 \mathrm{~kg}$ by subaxillary mini-thoracotomy. J Korean Med Sci. 2010 Jan; 25(1):24-7.

17. Clyman RI. Avery's diseases of the newborn. 9th ed. Philadelphia, PA: Elsevier Saunders; 2012. Capítulo 54, Patent ductus arteriosus in the premature infant; p. 751-61.

18. Shimada S, Kasai T, Konishi M, Fugiwara T, Effects of patent ductus arteriosus on left ventricular output and organ blood flows in preterm infants with respiratory distress syndrome treated with surfactant. J Pediatr. 1994;125(2):270-7.

19. Evans N. Diagnosis of patent ductus arteriosus in the preterm newborn. Arch Dis Child. Arch Dis Child. 1993;68:58-61.

20. Chaccur P, Dancini JL. Persistência do canal arterial. In: Santana MV, editor. Cardiopatias congênitas do recém-nascido: diagnóstico e tratamento. Rio de Janeiro: Atheneu; 2005. p. 272-5.

21. Metin K, Maltepe F, Kır M, Bilen Ç, Sökmen A, Oto O, et al.
Ligation of patent ductus arteriosus in low birth weight premature infants: timing for intervention and effectiveness of bed-side surgery. J Cardiothorac Surg. 2012;7:129.

22. Johnston PG, Gillam-Krakauer M, Fuller MP, Reese J. Evidence-Based Use of Indomethacin and Ibuprofen in the Neonatal Intensive Care Unit. Clin Perinatol. 2012;39(1):111-36.

23. Malviya MN, Ohlsson A, Shah SS. Surgical versus medical treatment with cyclooxygenase inhibitors for symptomatic patent ductus arteriosus in preterm infants. Cochrane Database Syst Rev. 2013;(3):CD003951

24. Heuchan AM, Clyman RI. Managing the patent ductus arteriosus: current treatment options. Arch Dis Child Fetal Neonatal Ed. 2014;99(5):F431-6.

25. Polit DF, Beck CT. Fundamentos de pesquisa em enfermagem: avaliação de evidências para a prática da enfermagem. 7 ed. Porto Alegre: Artmed; 2011. 669 p.

26. Conselho Nacional de Saúde (BR). Resolução n ${ }^{\circ}$ 466, de 12 de dezembro de 2012. DOU. 2013 jun 13;(112):59. Section 1.

27. Ramires JA, Filho RK. Cardiopatias congênitas: guia prático de diagnóstico, tratamento e conduta geral. Rio de Janeiro: Atheneu; 2014. Capítulo 19, Persistência do canal arterial; pag 287-300.

28. Miyague NI, Cardoso SM, Meyer F, Ultramari FT, Araujo FH, Rozkowisk I, et al. Estudo epidemiológico de cardiopatias congênitas na infância e adolescência. Análise em 4.538 casos. Arq Bras Cardiol. 2003;80(3):269-73.

29. Archer N. Patent ductus arteriosus in the newborn. Arch Dis Child. 1993;69 (5):529-32.

30. Rooberts D, Dalziel S. Antenatal corticosteroids for accelerating fetal lung maturation for women at risk of preterm birth. Cochrane Database Syst Rev. 2006 Jul 19;(3):CD004454.

\section{Como citar:}

Rabelo CM, Pinto VC Junior, Costa JE Filho, Castro EC, Aragão SS. Perfil dos recém-nascidos submetidos à correção cirúrgica de persistência do canal arterial à beira do leito. Rev Med UFC. 2017 jan-abr;57(1):19-24. 